\title{
Agenesis of the greater omentum with primary abdominal cocoon: experience of two cases
}

\author{
Ganguly $N N^{1}$, Bhattacharjee Nilutpal ${ }^{2}$, Bhoumick Rituparna ${ }^{3},{\text { Baruah } A R^{4}, \boldsymbol{R} \text { Anoop }}^{5}$
}

Received on October 30, 2019; Accepted on December 15, 2019

\begin{abstract}
Agenesis of the greater omentum along with primary abdominal cocoon is a rare condition. It is characterized by total or partial encasement of the small bowel inside a membranous bag. We report two cases of primary cocoon causing intestinal obstruction. The first case was encountered in the year 1998 and it was presented in a local conference as well as the Journal of the Association of the Surgeons of Assam. Long 21 years passed before we faced another case of agenesis of the greater omentum with primary abdominal cocoon causing acute dynamic small bowel obstruction. Both the cases were explored for unresolved obstruction and on table findings suggested primary abdominal cocoon. The findings were compared during the second case and the peculiarities were better noted, especially the agenesis of the greater omentum, presence of free peritoneal bodies. Photographs were taken.
\end{abstract}

Keywords: Intestinal obstruction; hypoplasia; agenesis of greater omentum.

\section{INTRODUCTION}

Agenesis of the greater omentum along with primary abdominal cocoon is a rare condition that refers to absence of the greater omentum from the transverse colon and along with total or partial containment of the small bowel inside a fibro-collagenous membrane bag leading to acute or chronic bowel obstruction. ${ }^{1,2}$ The cocoon has been described by various terms like 'peritonitis chronica fibrosa incapsulata' and sclerosing encapsulating peritonitis. We have encountered two patients who presented with features of acute intestinal obstruction due to abdominal cocoon and were treated successfully by surgery. Both the cases had agenesis of the greater omentum. Abdominal cocoon syndrome, which was first defined in $1978,{ }^{1}$ is relatively rare, with descriptions in the literature limited to case reports. In this syndrome, a portion or all of the small intestine is inside a bag like structure with a fibrocollagenous membrane not containing the mesothelium. As it is a rare entity, and its clinical findings are nonspecific, it is diagnosed during surgery. ${ }^{2}$ However, it can be diagnosed these days with contrastenhanced abdominal computerized tomography during the preoperative period. Surgical removal of the bag like structure and adhesiolysis are the treatment in these cases. This article presents a series of two patients with agenesis of the greater omentum with abdominal cocoon, who were successfully and surgically treated.

\section{MATERIALS AND METHODS}

Two cases were encountered over a period of 21 years.

First one a teenaged female, admitted with signs of acute dynamic intestinal obstruction. Plain X- Ray showed multiple gas fluid level in the pattern of small bowel obstruction. It failed to resolve on conservative treatment. Emergency exploration revealed a thick glistening membrane resembling a bag of peritoneum inside the peritoneal cavity. The bag had the whole of the small intestine inside. The terminal

Address for correspondence:

${ }^{1}$ Associate Professor (Corresponding author)

Email: drganguly@yahoo.com

Mobile: +919435017802

${ }^{2}$ Assistant Professor

${ }^{3-5}$ Post graduate student

Department of Surgery

Jorhat Medical College and Hospital, Jorhat, Assam, India.

Cite this article as: Ganguly NN, Bhattacharjee Nilutpal, Bhoumick Rituparna, Baruah AR, R Anoop. Agenesis of the greater omentum with primary abdominal cocoon: experience of two cases. Int J Health Res Medico Leg Prae 2020 January;6(1):7173. DOI 10.31741/ijhrmlp.v6.i1.2020.15 
ileum was obstructed by the edge of the bag like a sharp band. The large gut was empty and no obstruction was found. The greater omentum was severely hypoplastic to the amount of almost agenesis. The sac was excised totally. The obstruction relieved. No resection was needed. Recovery in the postoperative period was smooth and uneventful. No photographs could be kept in the midnight surgery, but operation records were kept. It was published in the local non indexed, non peer reviewed journal in 1998 (JASA).

The Second patient, a 40 years old healthy young adult male, presented with acute Intestinal obstruction and was opened up emergently. The same glistening membranous bag was detected (Figure 1). The extent of the membranous bag was similar to the first case. The severely Hypoplastic or agenesis of the greater omentum was documented with photographs this time (Figure 2). Two peritoneal free bodies (Peritoneal Mouse) incidentally were found (Figure 3). These were photographed and sent for histopathological examination. The sac excised totally. The patient recovered to be discharged normally. No resection of bowel was undertaken. Ultrasonography and CT scan of the whole abdomen failed to detect the cocoon before surgery.

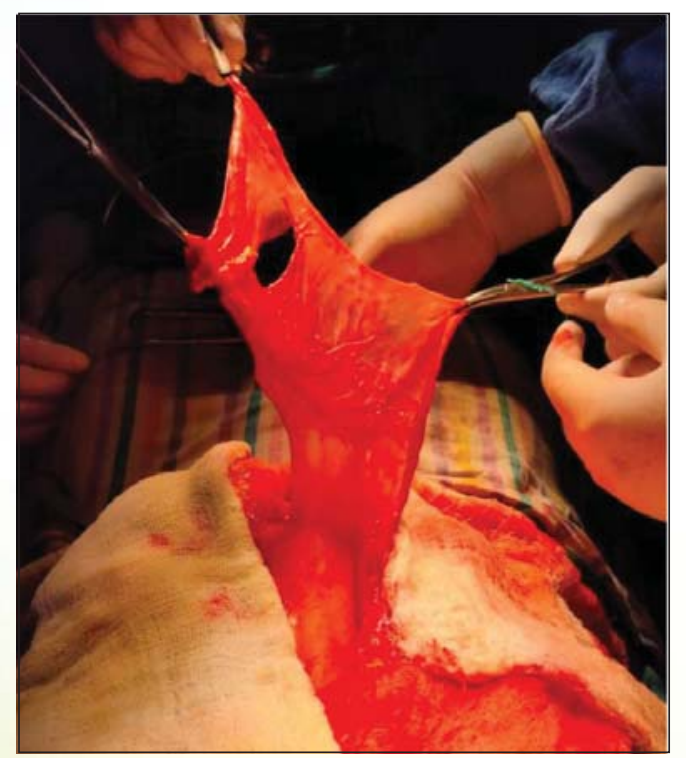

Figure 1 The Membranous bag

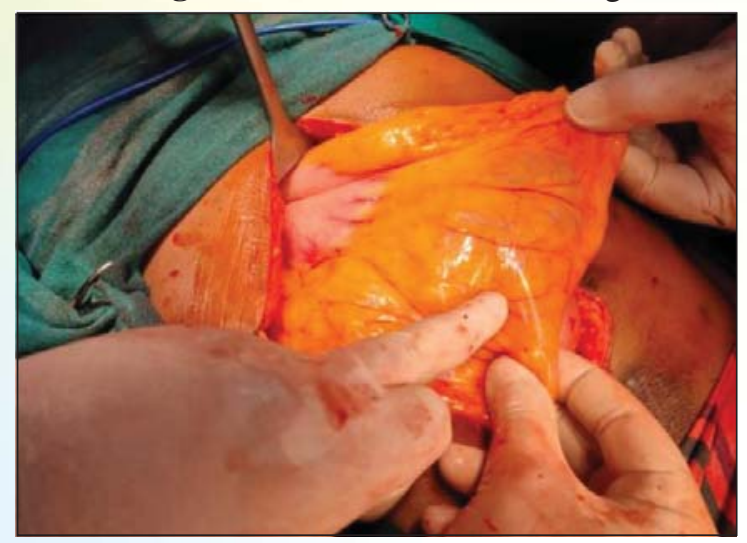

Figure 2 No Greater Omentum

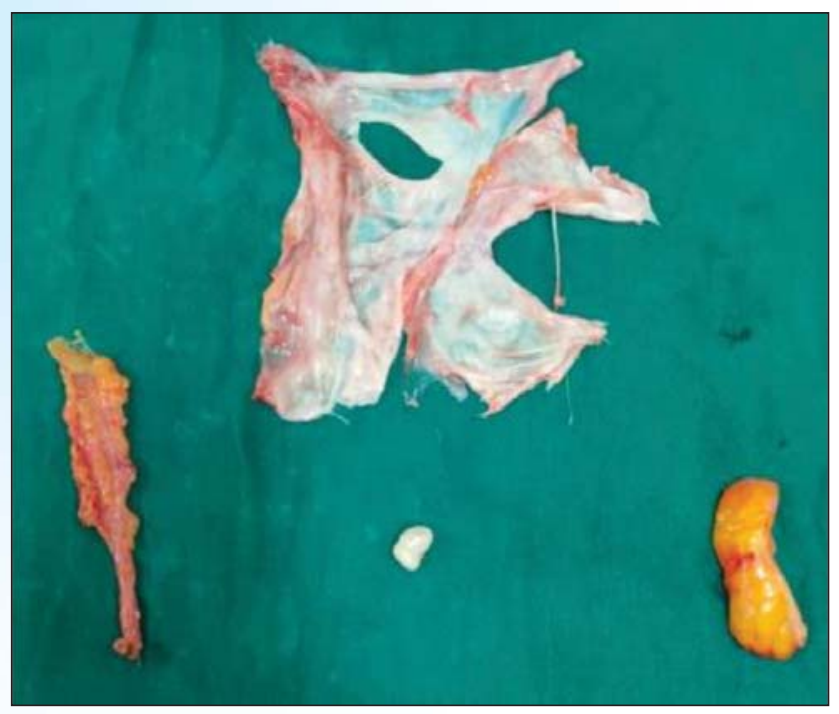

Figure 3 The Free peritoneal bodies with the dissected out cocoon ( $\mathrm{sac}$ )

Biopsy report from the membranous sac showed fibrous structure in a collagenous background. No evidence of any epithelium or mesothelium. The second case showed, both from the peritoneal mouse and the membrane, Fibrocollagenous tissue with occasional acute inflammatory cells. This too does not have any mesothelium. The pathologist suggested it to be samples of sclerosing encapsulating peritonitis.

\section{RESULTS}

Main clinical manifestations were complete intestinal obstruction in both the patients. The cases were diagnosed during exploratory emergency laparotomy. In both the cases, we found that most of the entire small bowel was encapsulated in a dense, glistening, smooth, white, fibrous, cocoon-like membrane, which looked like a bag of Peritoneum inside the peritoneal cavity. The greater omentum was absent in both cases. Excision of the membrane and release of adhesions were carefully performed to free the small intestine. Post-operative recovery in both the cases was smooth.

\section{DISCUSSION}

The agenesis of the greater omentum beyond the free border of the transverse colon could not be explained by the available embryological concepts. Abdominal cocoon however is classified as primary and secondary based on whether it is idiopathic or has a definite cause. The origin of the primary form is uncertain with various hypotheses, although it is probably caused by a subclinical peritonitis leading to the formation of a cocoon. ${ }^{1,3,4}$ Foo et al. detected the condition in 10 young girls with symptoms of intestinal obstruction two years post menarche.,5 It was postulated that a chemical peritonitis was caused by retrograde menstruation leading to the formation of a cocoon. ${ }^{4}$ Secondary causes include the placement of Le Veen shunts 
for refractory Ascitis, continuous ambulatory peritoneal dialysis, systemic lupus erythematosis, and the use of povidone iodine for abdominal wash-out, as well as the adrenergic blocker practolol. Practolol has been withdrawn from use because it was noted to cause the formation of a peritoneal membrane.

Clinically, patients with abdominal cocoon present with features of recurrent acute small bowel obstruction secondary to kinking and/ or compression of the intestines within the constricting cocoon. ${ }^{6}$ An abdominal mass may also be present due to an encapsulated cluster of dilated small bowel loops.

Abdominal X-ray findings are non-specific. CECT is considered an useful investigation for preoperative diagnosis of abdominal cocoon. The imaging features are, however, not confirmatory. CT findings of a membrane enveloping loops of small bowel were seen in some paraduodenal hernias, abdominal cocoon, and in peritoneal encapsulation. ${ }^{4}$ However, the clinical and pathological features of these entities are different.

The final diagnosis of abdominal cocoon is usually based on intra-operative and histopathology findings, with a significant number presenting for emergency treatment without any imaging being performed. In all the reported patients, portions of the small bowel were encased within a fibrous cocoon. ${ }^{7,8}$

Differential diagnosis also includes a condition called the peritoneal encapsulation, which is a developmental anomaly where the whole of the small bowel is encased in a thin accessory membrane. In peritoneal encapsulations, the patients are mostly asymptomatic and the findings are incidental and the patients present late in life. Treatment, as in the present case, consists of excision of the accessory peritoneal sac with lysis of the inter-loop adhesions. Bowel resection is unnecessary unless a nonviable segment is found.

In our two cases the hypoplasia or agenesis of the greater omentum was striking features along with the free peritoneal bodies. Other authors have also mentioned about this additional finding. ${ }^{7}$ The implications of these peculiar findings remain an enigma.

\section{CONCLUSION}

The clinical manifestation of Agenesis of the greater omentum along with abdominal cocoon is an enigma. We don't know the reasons of this association and could not explain the association. Abdominal cocoon otherwise is a specific and always present with intestinal obstruction of various stages. Preoperative diagnosis is difficult because of its rarity. The Agenesis of the greater omentum and the presence of the free peritoneal bodies present in our patients remain a mystery. The treatment of Abdominal Cocoon is surgery, and the recovery of our two patients was also satisfactory. As abdominal cocoon is a rare condition, diagnosis requires a high index of suspicion. Peritoneal sac excision, adhesiolysis and removal of the free bodies were the treatment and the outcome was satisfactory.

Conflict of interest: None declared.

Ethical clearance: Taken.

Source of funding: None declared.

Author disclosure: (1) The article is original with the author(s) and does not infringe any copyright or violate any other right of any third party. (2) The article has not been published (whole or in part) elsewhere and is not being considered for publication elsewhere in any form, except as provided herein. (3) All author(s) have contributed sufficiently in the article to take public responsibility for it and (4) all author(s) have reviewed the final version of the above manuscript and approved it for publication.

\section{REFERENCES}

1. Francis WY, Less SH. The abdominal cocoon. Aust N Z J Surg 1992;62:638-43.

2. Macklin J, Hall C, Feldman MA. Unusual cause of small bowel obstruction in adolescent girls: the abdominal cocoon. J R Coll Surg Edinb 1990;36:50-2.

3. Foo KT, Ng KC, Rauff A, Foong WC, Sinniah R. Unusual small intestinal obstruction in adolescent girls: the abdominal cocoon. Br J Surg 1978;65:427-30.

4. Seng LK, Mahadevan M, Musa A. Abdominal cocoon: a report of two cases. Br J Surg 1993;80:1149.

5. Ganguly NN. Primary abdominal cocoon; a case report. JASA 1998.

6. Wig JD, Goenka MK, Nagi B, Vaiphei K. Abdominal cocoon in a male: rare cause of intestinal obstruction. Trop Gastroenterol 1995;16:31-3.

7. Xiang Fei, Hai-Rui Yang, Peng-Fei Yu. Idiopathic abdominal cocoon syndrome with unilateral abdominal cryptorchidism and greater omentum hypoplasia in a young case of small bowel obstruction. World J Gastroenterol 2016;22(20):4958-62.

8. Ping $\mathrm{Xu}, \mathrm{Li}-\mathrm{Hua}$ Chen, You-Ming Li. Idiopathic sclerosing encapsulating peritonitis (or abdominal cocoon): a report of 5 cases. World J Gastroenterol 2007;13(26):3649-51. 